\title{
Voltage Stability of Weak Power Distribution Networks with Inverter Connected Sources
}

\author{
Zhao Wang ${ }^{1}$, Meng Xia ${ }^{1}$, and Michael Lemmon ${ }^{1}$
}

\begin{abstract}
Microgrids are power distribution systems in which generation is located close to loads. These small distribution networks represent a bottom-up approach for improving the power security of critical loads that cannot tolerate disruptions in main grid service. Voltage stability becomes a significant issue in microgrids when the network interconnections are weak. This paper derives sufficient conditions for voltage stability of a weak microgrid with inverter connected sources. These conditions take the form of inequality constraints on various network parameters, loads and generation setpoints. These conditions can therefore be easily incorporated into dispatch optimization problems.
\end{abstract}

\section{INTRODUCTION}

Microgrids are small-scale power distribution systems in which generation sources are located close to loads. These power networks represent a bottom-up approach to improve the security of power delivery that focuses on enhancing generation capacity at the distribution level of a national power grid. This approach encourages consumption of locally generated power in a way that reduces peak loads seen by distribution network operators (DSOs). This method reduces the demand for extra transmission capacity and provides a path for greater use of renewable energy resources. Microgrids can also be operated independently from the main grid (also known as islanded mode) thereby making them invaluable for safety-critical loads that cannot tolerate main grid power outages (e.g. hospitals), that may be disconnected from the grid unexpectedly (e.g. military bases), and areas in which a national main grid has yet to penetrate (e.g. rural electrification). Microgrids therefore represent an important power distribution technology whose safe and efficient operation can significantly secure the delivery of power to millions of users.

As small-scale distribution networks, the links in microgrids can be weak. The cables interconnecting the buses often have higher ratios between their conductance and susceptance, $G / B$, than those found in transmission networks. A consequence of this higher ratio is that dynamics governing voltage and phase are more highly coupled, thereby making it more difficult to guarantee voltage stability [18].

There has, of course, been a great deal of prior work studying voltage stability in such weak networks [4][12][18][21]. Most of those efforts, however, have studied specific network interconnect topologies with simplifying assumptions, such

\footnotetext{
*This work was supported by the National Science Foundation (CNS0931195).

${ }^{1}$ Zhao Wang, Meng Xia and Michael Lemmon are with the department of Electrical Engineering, University of Notre Dame, Notre Dame, IN 46556, USA. zwang6, mxia, lemmon at nd.edu
}

as parallel [4] or chain [21] structures. Previous research efforts include both linearization and Lyapunov approaches. In linearization methods, eigenvalue analysis only applies locally [2] [11] [20] [12] [16] [18] [21]. In contrast, Lyapunov methods have been used to assess global stability properties [3], but the conditions derived are usually hard to check. It is therefore unclear how thus prior work leads to an analytic set of constraints whose satisfaction assure stability over a wide class of weak distribution networks.

The objective of this paper is to develop constraints whose satisfaction assure voltage stability of CERTS (Consortium for Electric Reliability Technology Solutions) microgrids. CERTS microgrids [13] represent an important class of microgrid that use fast inverters to connect a heterogenous mixture of generation sources into the network. Earlier work [11] has studied the stability of relatively strong CERTS microgrids, but to our best knowledge there has been little work examining stability conditions for weak networks. Recent work [7] has attempted to address that issue by viewing the network as a set of coupled nonlinear oscillators. Building upon that prior work, this paper derives a set of pointwise inequality constraints whose satisfaction assures voltage stability and frequency synchronization in islanded CERTS microgrids. These stability conditions place constraints on interconnection weakness, requested power generation, and reactive loads. When combined with optimal dispatch problems, these constraints provide a systematic framework for optimal distributed generation dispatch and demand side management in this important class of microgrid.

The remainder of this paper is organized as follows. Section II reviews the power system background and notational conventions used throughout the paper. Section III presents the weak network model. Section IV presents the main results of the paper. Section V shows how this paper's stability constraints can be directly incorporated into an optimal dispatch problem. Section VI consists of simulation examples illustrating the conservativeness of our stability condition. Section VII provides concluding remarks and identifies future directions in realizing stable and optimal power dispatch.

\section{BACKGROUND AND NOTATIONS}

This section presents basic definitions and notations that are used throughout this paper. All system states are expressed with per unit (p.u.) normalization. This paper also assumes balanced three-phase operations, so that per phase circuit models are implemented. With per phase circuit 
models, the analysis in this paper also applies to single-phase power networks.

Consider a distribution network consisting of $n$ buses and let $Y_{n \times n}$ be a symmetric complex valued $n \times n$ matrix representing the network's admittance matrix. The $i j$ th component of $Y_{n \times n}$ may be expressed as

$$
\begin{aligned}
& Y_{i j}=\left\{\begin{array}{cc}
-\frac{1}{Z_{i j}} & \text { if bus } \mathrm{i} \text { and } \mathrm{j} \text { are connect, } \\
0 & \text { else, }
\end{array}\right. \\
& Y_{i i}=\sum_{j=1, j \neq i}^{n}-Y_{i j},
\end{aligned}
$$

where $Z_{i j}$ is the impedance between bus $i$ and $j$ for all $i, j \in\{1,2, \ldots, n\} . Y_{n \times n}$ may also be expressed as $Y_{n \times n}=$ $G_{n \times n}+j B_{n \times n}$, where $G_{n \times n}$ is the conductance matrix and $B_{n \times n}$ is the susceptance matrix. For elements of the two matrices $\left\{G_{i j}\right\}$ and $\left\{B_{i j}\right\}$, where $i, j \in\{1,2, \ldots, n\}$ : if $j=i$, then $G_{i i}>0$ and $B_{i i}<0$; if $j \neq i$, there are $G_{i j}=G_{j i} \leq 0$ and $B_{i j}=B_{j i} \geq 0$.

The complex power injected into bus $i$ is denoted as $S_{i}=$ $P_{i}+j Q_{i}(i=1,2, \ldots, n)$, where $P_{i}$ is real power and $Q_{i}$ is reactive power. At bus $i, S_{i}$ satisfies

$$
S_{i}=3 V_{i} I_{i}^{*}=3 V_{i}\left(\sum_{j=1}^{n} Y_{i j} V_{j}\right)^{*}
$$

which may be rewritten in matrix-vector form as $\vec{S}=$ $3 \vec{V} \vec{I}^{*}=3 \vec{V}\left(Y_{n \times n} \vec{V}\right)^{*} \cdot \vec{V}, \vec{I} \in \mathbb{C}$ are $n$-dimensional complex vectors whose $i$ th components are $V_{i}$, the voltage at bus $i$ relative to ground, and $I_{i}$, the current entering bus $i$, respectively.

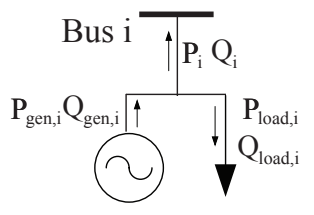

Fig. 1. Power Balance at Bus i

As demonstrated in Figure 1, positive $P_{i}$ and $Q_{i}$ inject power flows into bus $i . P_{g e n, i}$ and $Q_{g e n, i}$ describe the real and reactive power generated at bus $i$. $P_{l o a d, i}$ and $Q_{l o a d, i}$ denote the collection of real and reactive powers absorbed by all loads at bus $i$. Constant impedance load values are defined at the nominal voltage, so that the loads are proportional to the square of voltage magnitudes $\left\{E_{i}\right\}$. Therefore the injected real and reactive powers into bus $i$ are

$$
\begin{aligned}
P_{i} & =P_{\text {gen }, i}-E_{i}^{2} P_{\text {load }, i}, \\
Q_{i} & =Q_{\text {gen }, i}-E_{i}^{2} Q_{\text {load }, i} .
\end{aligned}
$$

The dependence of $P_{i}$ and $Q_{i}$ on neighboring buses is captured by the power balance relations

$$
\begin{aligned}
P_{i} & =3 \sum_{j=1}^{n} E_{i} E_{j}\left(G_{i j} \cos \left(\delta_{i}-\delta_{j}\right)+B_{i j} \sin \left(\delta_{i}-\delta_{j}\right)\right), \\
Q_{i} & =3 \sum_{j=1}^{n} E_{i} E_{j}\left(G_{i j} \sin \left(\delta_{i}-\delta_{j}\right)-B_{i j} \cos \left(\delta_{i}-\delta_{j}\right)\right),
\end{aligned}
$$

where $E_{i}$ and $\delta_{i}$ are the voltage magnitude and the phase angle at bus $i$. Given the real powers $\left\{P_{i}\right\}$ and voltage magnitudes $\left\{E_{i}\right\}$, one can use equations (4-5) to solve for the corresponding reactive powers $\left\{Q_{i}\right\}$ and phase angles $\left\{\delta_{i}\right\}$. Real valued vectors $(P, Q, \delta, E)$ are time-variant signals of system states. Existence and uniqueness of solution to this power flow analysis rest upon the Lipschitz nature of these power balance relations [9].

\section{Problem Statement}

This section presents the CERTS Microgrid system models. As shown in Figure 2, an islanded microgrid may be viewed as the interconnection of four subsystems, including the voltage control block, the frequency synchronization block, and two power balance blocks. The controller blocks use droop mechanisms that down-scale conventional grid control concepts to low voltage power grids [8]. The particular controller designs are based on the CERTS droop controllers [13].

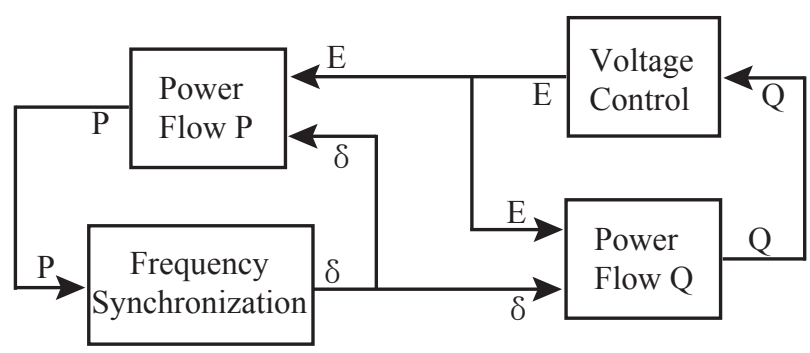

Fig. 2. Complete Model of the Network

The dynamics are generated by those local controllers tied to each bus, with the power balance relations in equations (4-5). The associated controller equations are therefore

$$
\begin{aligned}
\dot{\delta}_{i} & =m_{P}\left(P_{i}^{*}-P_{g e n, i}\right)+\omega_{0}, \\
& =m_{P}\left(P_{i}^{*}-P_{i}-E_{i}^{2} P_{l o a d, i}\right)+\omega_{0}, \\
\dot{E}_{i} & =K_{Q}\left(E_{i}^{*}-E_{i}\right)-m_{Q} Q_{g e n, i} \\
& =K_{Q}\left(E_{i}^{*}-E_{i}\right)-m_{Q}\left(Q_{i}+E_{i}^{2} Q_{\text {load }, i}\right),
\end{aligned}
$$

for all $i \in\{1,2, \ldots, n\}$. Equations (6-7) are the frequency and voltage controls, respectively, for the $i$ th bus. These equations describe how the real and reactive powers injected at bus $i$ are modulated based on the voltage magnitude $E_{i}$ and phase angle $\delta_{i}$ on this bus.

In equations (6-7), $P_{i}^{*}$ and $E_{i}^{*}$ denote the operator's commanded real power and voltage levels at the $i$ th bus. The parameters $m_{P}, K_{Q}$, and $m_{Q}$, are droop control parameters, while $\omega_{0}$ is the desired angular frequency $\left(120 \pi \mathrm{rad} \cdot \mathrm{s}^{-1}\right)$.

Set point $\left(P_{\text {set }}, Q_{\text {set }}, \delta_{\text {set }}, E_{\text {set }}, \omega_{\text {set }}\right)$ is an equilibrium point of the system in equations (4-7), where $\omega_{\text {set }}$ is the corresponding system frequency of the set point. Given $\left\{P_{i}^{*}\right\}$ and $\left\{E_{i}^{*}\right\}$, the set point is obtained by solving equations (45) for all $i \in\{1,2, \ldots, n\}$ with the help of the following relationships obtained by zeroing the time derivatives in 
equations (6-7)

$$
\begin{aligned}
& 0=m_{P}\left(P_{i}^{*}-P_{\text {set }, i}-E_{\text {set }, i}^{2} P_{\text {load }, i}\right)+\omega_{0}, \\
& 0=K_{Q}\left(E_{i}^{*}-E_{\text {set }, i}\right)-m_{Q}\left(Q_{\text {set }, i}+E_{\text {set }, i}^{2} Q_{\text {load }, i}\right) .
\end{aligned}
$$

The voltage stability of this system is studied using phase angle, voltage and reactive power errors defined as

$$
\tilde{\delta}=\delta-\delta_{\text {set }}, \tilde{E}=E-E_{\text {set }}, \tilde{Q}=Q_{\text {set }}-Q .
$$

Based on equation (9), the operator commanded $E_{i}^{*}$ satisfies

$$
E_{i}^{*}=E_{\text {set }, i}+\frac{m_{Q}}{K_{Q}}\left(Q_{\text {set }, i}+E_{\text {set }, i}^{2} Q_{\text {load }, i}\right) .
$$

The voltage control block in Figure 2 treats $\left\{Q_{i}\right\}$ as the input and $\left\{E_{i}\right\}$ as the output. The associated error equation therefore take the form of

$$
\begin{aligned}
\dot{\tilde{E}}_{i}= & \dot{E}_{i}-\dot{E}_{\text {set }, i} \\
= & K_{Q}\left(E_{i}^{*}-E_{i}\right)-m_{Q}\left(Q_{i}+E_{i}^{2} Q_{\text {load }, i}\right) \\
= & K_{Q}\left(E_{\text {set }, i}-E_{i}\right)+m_{Q}\left(Q_{\text {set }, i}-Q_{i}\right) \\
& +m_{Q}\left(E_{\text {set }, i}^{2}-E_{i}^{2}\right) Q_{\text {load }, \text { nom }, i} \\
= & m_{Q} \tilde{Q}_{i}-\left[K_{Q}+m_{Q}\left(2 E_{\text {set }, i}+\tilde{E}_{i}\right) Q_{\text {load }, i}\right] \tilde{E}_{i} .
\end{aligned}
$$

Definition 1: System in equations (6-7) have voltage stability if: the inputs $P, P_{\text {load }}, Q$, and $Q_{\text {load }}$ are constant, there are two open subsets of $\Omega_{E, 1}, \Omega_{\delta, 1} \subset \mathbb{R}^{n}$ containing the origin such that if $\tilde{E}(0) \in \Omega_{E, 1}$ and $\delta(0) \in \Omega_{\delta, 1}$ then $\tilde{E}(t) \rightarrow 0$ as $t \rightarrow \infty$.

Lemma 1 and 2 bound the value of $\tilde{Q}$. Proofs of these lemmas are provided in the Appendix.

Lemma 1: Defined $l_{E}$ and $l_{\delta}$ as

$$
\begin{aligned}
l_{E} & =6\left(\max _{i}\left(E_{\text {set }, i}\right)+\left|\tilde{E}_{i}\right|_{\max }\right)\left(\left|G_{i i}\right|_{\max }+2\left|B_{i i}\right|_{\max }\right), \\
l_{\delta} & =6\left(\max _{i}\left(E_{\text {set }, i}\right)+\left|\tilde{E}_{i}\right|_{\max }\right)^{2}\left(\left|G_{i i}\right|_{\max }+\left|B_{i i}\right|_{\max }\right),
\end{aligned}
$$

$\left|\tilde{Q}_{i}\right|$ is bounded by

$$
\begin{aligned}
\left|\tilde{Q}_{i}\right| & \leq l_{E}\left|\tilde{E}_{i}\right|_{\max }+l_{\delta}\left|\tilde{\delta}_{i}\right|_{\max }, \\
& \leq l_{E}\left|\tilde{E}_{i}\right|_{\max }+l_{\delta} \cdot 2 \pi .
\end{aligned}
$$

Lemma 2: Define $m_{E}$ and $m_{\delta}$ as $m_{E}=\max \left\{\sqrt{\lambda}: \lambda\right.$ is an eigenvalue of $\left.\left(\frac{\partial Q}{\partial E}\right)^{*}\left(\frac{\partial Q}{\partial E}\right)\right\}$, $m_{\delta}=\max \left\{\sqrt{\lambda}: \lambda\right.$ is an eigenvalue of $\left.\left(\frac{\partial Q}{\partial \delta}\right) *\left(\frac{\partial Q}{\partial \delta}\right)\right\}$

then $\|\tilde{Q}\|_{2}$ is bounded by

$$
\|\tilde{Q}\|_{2} \leq m_{E}\|\tilde{E}\|_{2}+\sqrt{n} m_{\delta}\left|\tilde{\delta}_{i}\right|_{\max }
$$

By inserting the power balance relation (4) into the earlier droop equation (6), the frequency controller is written as

$$
\begin{aligned}
\dot{\delta}_{i}= & m_{P}\left(P_{i}^{*}-P_{\text {gen }, i}\right)+\omega_{0}, \\
= & m_{P}\left(P_{i}^{*}-P_{i}-E_{i}^{2} P_{\text {load }, i}\right)+\omega_{0}, \\
= & \omega_{0}+m_{P}\left(P_{i}^{*}-E_{i}^{2} G_{i i}-E_{i}^{2} P_{\text {load }, i}\right) \\
& -3 m_{P} \sum_{\substack{j=1 \\
j \neq i}}^{n} E_{i} E_{j}\left(G_{i j} \cos \left(\delta_{i}-\delta_{j}\right)+B_{i j} \sin \left(\delta_{i}-\delta_{j}\right)\right), \\
= & \omega_{i}-m_{P} \sum_{\substack{j=1 \\
j \neq i}}^{n} 3 E_{i} E_{j}\left|Y_{i j}\right| \sin \left(\delta_{i}-\delta_{j}+\phi_{i j}\right),
\end{aligned}
$$

where the natural frequency is $\omega_{i}=\omega_{0}+m_{P}\left(P_{i}^{*}-3 E_{i}^{2} G_{i i}-\right.$ $\left.E_{i}^{2} P_{\text {load }, i}\right) ; \phi_{i j}$ is the phase shift associated with the link between bus $i$ and $j, \phi_{i j}=\phi_{j i}=\tan ^{-1}\left(\frac{G_{i j}}{B_{i j}}\right) \in\left[-\frac{\pi}{2}, 0\right]$; the diagonal terms are $\left|Y_{i i}\right|=0$ and $\phi_{i i}=0$.

Buses in a power network are treated as interconnected nonlinear oscillators [5] [6]. Synchronization of these oscillators corresponds to frequency synchronization of the power network [1].

Definition 2: System in equations (6-7) have frequency synchronization: the inputs $P, P_{\text {load }}, Q$, and $Q_{\text {load }}$ are constant, there are two open subsets of $\Omega_{E, 2}, \Omega_{\delta, 2} \subset \mathbb{R}^{n}$ containing the origin such that if $\tilde{E}(0) \in \Omega_{E, 2}$ and $\delta(0) \in$ $\Omega_{\delta, 2}$ then $\dot{\delta}(t) \rightarrow \dot{\delta}_{\infty}=\omega_{\text {set }}$ as $t \rightarrow \infty$.

In conclusion, equations (4-5), (11), and (14) form the set of state equations for the system being investigated, which is shown in Figure 2.

\section{Main Result}

This section derives sufficient conditions for voltage stability and frequency synchronization in inverter based CERTS microgrids. The result consists of two steps. This paper first identifies positively invariant sets for voltage magnitudes $\left\{E_{i}\right\}$, and phase angles, $\left\{\delta_{i}\right\}$ where $i \in\{1,2, \ldots, n\}$. Assuming that the system's initial states start within these invariant sets, we first establish sufficient conditions for phase synchronization and then establish the network's voltage stability.

\section{A. Invariant Sets}

The following lemma characterizes a positively invariant set of voltage magnitudes.

Lemma 3: Consider the system model described by equations $(4-5,11,14)$, and set $\left|\tilde{\delta}_{i}\right|$ to be the maximum possible value of $2 \pi$ for any bus $i$, given

$$
\begin{aligned}
\left|Q_{\text {load }, i}\right|_{\max }< & a \\
K_{Q} / m_{Q}> & \max \left(b_{1}+2 \sqrt{\left(-Q_{\text {load }, i}+a\right) c}\right. \\
& -b_{2}+2 \sqrt{\left.\left(Q_{\text {load }, i}+a\right) c\right)}
\end{aligned}
$$

where

$$
\begin{aligned}
a= & (6+12 \pi)\left|G_{i i}\right|_{\text {max }}+(12+12 \pi)\left|B_{\text {ii }}\right|_{\text {max }}, \\
b_{1}= & \left((6+24 \pi)\left|G_{i i}\right|_{\text {max }}+(12+24 \pi)\left|B_{i i}\right|_{\text {max }}\right. \\
& \left.-2 Q_{\text {load }, i}\right) \max _{i}\left\{E_{\text {set }, i}\right\}, \\
b_{2}= & \left((6+24 \pi)\left|G_{i i}\right|_{\text {max }}+(12+24 \pi)\left|B_{\text {ii }}\right|_{\max }\right. \\
& \left.+2 Q_{\text {load }, i}\right) \max _{i}\left\{E_{\text {set }, i}\right\}, \\
c= & 12 \pi\left(\left|G_{\text {ii }}\right|_{\text {max }}+\left|B_{\text {ii }}\right|_{\text {max }}\right)\left(\max _{i}\left\{E_{\text {set }, i}\right\}\right)^{2} .
\end{aligned}
$$

If $\max _{i} E_{i} \leq E_{\max }=\max _{i}\left(E_{\text {set }, i}\right)$

$$
+\frac{K_{Q} / m_{Q}-b_{1}+\sqrt{\left(b_{1}-K_{Q} / m_{Q}\right)^{2}-4\left(a-Q_{\text {load }, i}\right) c}}{2\left(a-Q_{\text {load }, i}\right)},
$$

and $\min _{i} E_{i} \geq E_{\text {min }}=\min _{i}\left(E_{\text {set }, i}\right)$

$$
-\frac{K_{Q} / m_{Q}+b_{2}+\sqrt{\left(K_{Q} / m_{Q}+b_{2}\right)^{2}-4\left(Q_{\text {load }, i}+a\right) c}}{2\left(Q_{\text {load }, i}+a\right)},
$$


then the set $\mathcal{I}_{E}$, defined as

$\mathcal{I}_{E}=\left\{E \in \mathbb{R}^{n}: E_{\min } \leq E_{i} \leq E_{\max }, 0<E_{\min }<E_{\max }\right\}$,

is a positively invariant set with respect to the system equation (11).

Proof: $\mathcal{I}_{E}$ will be an invariant set, if, for arbitrary $i \in\{1,2, \ldots n\},\left|\tilde{E}_{i}\right|$ is non-increasing on the border of $\mathcal{I}_{E}$. There are two cases to consider in the analysis, when $E_{i}=E_{\max }$, then $\tilde{E}_{i}>0$. Inserting equation (12) into equation (11) yields

$$
\begin{aligned}
& \dot{\tilde{E}}_{i} \leq-\left[K_{Q}+m_{Q}\left(2 E_{\text {set }, i}+\tilde{E}_{i}\right) Q_{\text {load }, i}\right] \tilde{E}_{i} \\
& +6 m_{Q}\left(E_{\text {set }, i}+\tilde{E}_{i}\right)\left(\left|G_{i i}\right|_{\text {max }}+2\left|B_{i i}\right|_{\text {max }}\right) \tilde{E}_{i} \\
& +6 m_{Q}\left(E_{\text {set }, i}+\tilde{E}_{i}\right)^{2}\left(\left|G_{i i}\right|_{\text {max }}+\left|B_{i i}\right|_{\text {max }}\right) \cdot 2 \pi, \\
= & m_{Q}\left[\left(-Q_{\text {load }, i}+a\right) \tilde{E}_{i}^{2}+\left(-K_{Q} / m_{Q}+b_{1}\right) \tilde{E}_{i}+c\right] .
\end{aligned}
$$

$\dot{\tilde{E}}_{i}$ should be non-positive to make $\tilde{E}_{i}>0$ not increasing. Given the lemma's hypothesis (16), the equation $\left(-Q_{\text {load }, i}+\right.$ a) $x^{2}+\left(-K_{Q} / m_{Q}+b_{1}\right) x+c=0$ has two real solutions, at least one of them being positive. If (22) is satisfied, then $\tilde{E}_{i}$ is non-increasing on the border of $E_{i}=E_{\max }$.

The other case occurs when $E_{i}=E_{\text {min }}$, then $\tilde{E}_{i}<0$

$$
\begin{aligned}
& \dot{\tilde{E}}_{i} \geq-\left[K_{Q}+m_{Q}\left(2 E_{\text {set }, i}+\tilde{E}_{i}\right) Q_{\text {load }, i}\right] \tilde{E}_{i} \\
& -6 m_{Q}\left(E_{\text {set }, i}+\tilde{E}_{i}\right)\left(\left|G_{i i}\right|_{\text {max }}+2\left|B_{i i}\right|_{\text {max }}\right) \tilde{E}_{i} \\
& -6 m_{Q}\left(E_{\text {set }, i}+\tilde{E}_{i}\right)^{2}\left(\left|G_{i i}\right|_{\text {max }}+\left|B_{i i}\right|_{\text {max }}\right) \cdot 2 \pi, \\
= & -m_{Q}\left[\left(Q_{\text {load }, i}+a\right) \tilde{E}_{i}^{2}+\left(K_{Q} / m_{Q}+b_{2}\right) \tilde{E}_{i}+c\right] .
\end{aligned}
$$

$\dot{\tilde{E}}_{i}$ should be non-negative to make $\tilde{E}_{i}<0$ not decreasing. Given the lemma's hypothesis (16), the equation $\left(Q_{\text {load }, i}+\right.$ a) $x^{2}+\left(K_{Q} / m_{Q}+b_{2}\right) x+c=0$ has two real solutions, at least one of them being negative. If (23) is satisfied, then $\tilde{E}_{i}$ is non-decreasing on the border of $E_{i}=E_{\text {min }}$.

For any $i \in\{1,2, \ldots, n\}$ when $E_{i}=E_{\min }$ or $E_{\max },\left|\tilde{E}_{i}\right|$ does not increase, hence any $E_{i}$ stays in $\mathcal{I}_{E}$ once it starts between $E_{\min }$ and $E_{\max }$. Therefore, the two conditions in (21-22) imply that $\mathcal{I}_{E}$ is positively invariant.

Remark 1: Equation (15) restricts the reactive load $Q_{\text {load }, i}$ by $a$. This requirement relates to the coupling strength of the network through $\left|G_{i i}\right|_{\max }$ and $\left|B_{i i}\right|_{\max }$.

Remark 2: Equation (16) requires the ratio $K_{Q} / m_{Q}$ to be large enough, which physically demonstrates the control force of the inverter. A too large ratio may bring difficulties to the construction of these inverters.

The following lemma derives a positively invariant set of phase shifts $\left\{\delta_{i}\right\}$. The analysis draws upon techniques used in [5][7].

Lemma 4: Assume the conditions of lemma 3 are satisfied. Define $A_{1}$ and $A_{2}$ as

$$
\begin{aligned}
A_{1}= & 3 n E_{\min }^{2} \min _{i \neq j}\left|B_{i j}\right|, \\
A_{2}= & \max _{i \neq j}\left(\left|P_{\text {set }, i}-P_{\text {set }, j}\right|+3 E_{\max }^{2}\left|G_{i i}-G_{j j}\right|\right) \\
& -6 E_{\min }^{2}\left|G_{i i}\right|_{\min },
\end{aligned}
$$

where $E_{\min }$ and $E_{\max }$ are from $\mathcal{I}_{E}$. If

$$
A_{1} \sin (\theta) \geq A_{2},
$$

then there exists a non-empty set $\mathcal{I}_{\theta}$

$$
\mathcal{I}_{\theta}=\left\{\delta \in \mathbb{R}^{n}: \max _{i, j}\left|\delta_{i}-\delta_{j}\right| \leq \theta, \theta \in[0, \pi]\right\},
$$

which is positively invariant with respect to system equations (4-5,11,14).

Proof: Define a positive function $V_{\delta}(\delta): \mathbb{R}^{n} \rightarrow[0, \pi]$ for the network with $n$ buses as

$$
V_{\delta}(\delta)=\frac{1}{m_{P}} \max _{i \neq j}\left\{\left|\delta_{i}-\delta_{j}\right|\right\}=\frac{1}{m_{P}}\left(\delta_{k}-\delta_{l}\right),
$$

where $\delta_{k}$ achieves clockwise maximum and $\delta_{l}$ achieves the counterclockwise minimum, with $k, l \in\{1,2, \ldots, n\}$. Assume that $\left|\delta_{i}(0)-\delta_{j}(0)\right| \leq \theta$ for any $i, j \in\{1,2, \ldots, n\}$, where $\theta$ is arbitrary and $\theta \in[0, \pi]$, such that all angles are contained in an arc of length $\theta$.

Taking the upper Dini derivative of $V_{\delta}, D^{+} V_{\delta}$ is

$$
\begin{aligned}
& \dot{\delta}_{k}-\dot{\delta}_{l} \\
= & \left(P_{s e t, k}-P_{s e t, l}\right)-3\left(E_{k}^{2} G_{k k}-E_{l}^{2} G_{l l}\right) \\
- & 3\left[\sum_{\substack{j=1 \\
j \neq k}}^{n} E_{k} E_{j}\left(G_{k j} \cos \left(\delta_{k}-\delta_{j}\right)+B_{k j} \sin \left(\delta_{k}-\delta_{j}\right)\right)\right. \\
- & \left.\sum_{\substack{j=1 \\
j \neq l}}^{n} E_{l} E_{j}\left(G_{l j} \cos \left(\delta_{l}-\delta_{j}\right)+B_{l j} \sin \left(\delta_{l}-\delta_{j}\right)\right)\right] \\
= & \left(P_{s e t, k}-P_{s e t, l}\right)-3\left(E_{k}^{2} G_{k k}-E_{l}^{2} G_{l l}\right) \\
- & 3\left[\sum_{\substack{j=1 \\
j \neq k}}^{n} E_{k} E_{j} B_{k j} \sin \left(\delta_{k}-\delta_{j}\right)-\sum_{\substack{j=1 \\
j \neq l}}^{n} E_{l} E_{j} B_{l j} \sin \left(\delta_{l}-\delta_{j}\right)\right] \\
- & 3\left[\sum_{\substack{j=1 \\
j \neq k}}^{n} E_{k} E_{j} G_{k j} \cos \left(\delta_{k}-\delta_{j}\right)-\sum_{\substack{j=1 \\
j \neq l}}^{n} E_{l} E_{j} G_{l j} \cos \left(\delta_{l}-\delta_{j}\right)\right] .
\end{aligned}
$$

From $V(\delta) \leq \theta$, there are $\left(\delta_{k}-\delta_{j}\right) \in[0, \theta]$ and $\left(\delta_{j}-\delta_{l}\right) \in$ $[0, \theta]$, so that $\sin \left(\delta_{k}-\delta_{j}\right) \geq 0$ and $\sin \left(\delta_{j}-\delta_{l}\right) \geq 0$. Moreover, the following inequality holds,

$$
\begin{aligned}
& \sin \left(\delta_{k}-\delta_{j}\right)+\sin \left(\delta_{j}-\delta_{l}\right) \\
= & 2 \sin \left(\frac{\delta_{k}-\delta_{l}}{2}\right) \cos \left(\frac{\delta_{k}+\delta_{l}}{2}-\delta_{j}\right) \\
\geq & 2 \sin \frac{\theta}{2} \cos \frac{\theta}{2}=\sin \theta .
\end{aligned}
$$

Replacing the sum of sinusoidal functions with its lower bound $\sin \theta$, there is

$$
\begin{aligned}
D^{+} V_{\theta}= & \left(P_{k}^{*}-P_{l}^{*}\right)-\left(P_{k}-P_{l}\right) \\
\leq & \left(P_{\text {set }, k}-P_{\text {set }, l}\right)-3\left(E_{k}^{2} G_{k k}-E_{l}^{2} G_{l l}\right) \\
& -3 E_{\min }^{2} n \min _{i \neq j} B_{i j} \sin \theta \\
& -6 E_{\min }^{2}\left|G_{i i}\right|_{\text {min }} \\
\leq & \max _{i \neq j}\left[\left|P_{\text {set }, i}-P_{\text {set }, j}\right|+3 E_{\max }^{2}\left|G_{i i}-G_{j j}\right|\right] \\
& -3 E_{\min }^{2} \min _{i \neq j} n B_{i j} \sin (\theta)-6 E_{\min }^{2}\left|G_{i i}\right|_{\text {min }} .
\end{aligned}
$$

Under the lemma's condition, $A_{1} \sin \theta \geq A_{2}$. It should be clear that $D^{+} V_{\theta} \leq 0$ and therefore $V_{\theta}$ is non-increasing. As a result, $\mathcal{I}_{\theta}$ is a positively invariant set. 
Remark 3: Condition $A_{1} \sin (\theta) \geq A_{2}$ will be satisfied if $\left|B_{i j}\right|$ is sufficiently greater than $\left|G_{i j}\right|$. This is, essentially a limit on network weakness.

\section{B. Asymptotic Stability}

The following theorem establishes sufficient conditions for frequency synchronization.

Theorem 3: Under conditions in lemma 3 and 4, if

$$
A_{1} \sin \left(\pi / 2-\alpha_{\max }\right) \geq A_{2},
$$

where $\alpha_{\max }=\max _{i j} \tan ^{-1}\left(-G_{i j} / B_{i j}\right)$, then frequency $\dot{\delta}_{i}(t) \rightarrow \dot{\delta}_{\infty}=\omega_{\text {set }}$ as $t \rightarrow \infty$, for all $i \in\{1,2, \ldots, n\}$.

Proof: Define $\alpha_{i j}=-\phi_{i j}=\tan ^{-1}\left(-\frac{G_{i j}}{B_{i j}}\right) \in\left[0, \frac{\pi}{2}\right]$. Take bus voltages as inputs, derivatives of equation (11) is

$$
\frac{d}{d t} \dot{\delta}_{i}=-3 \sum_{\substack{j=1 \\ j \neq i}}^{n} E_{i} E_{j}\left|Y_{i j}\right| \cos \left(\delta_{i}-\delta_{j}-\alpha_{i j}\right)\left(\dot{\delta}_{i}-\dot{\delta}_{j}\right),
$$

rewritten in a vector form,

$$
\frac{d}{d t} \dot{\delta}=\vec{F}(t) \dot{\delta}
$$

where $F(t)$ is a time-varying matrix whose components are

$$
\begin{aligned}
& F_{i i}=-3 m_{P} \sum_{\substack{j=1 \\
j \neq i}}^{n} E_{i} E_{j}\left|Y_{i j}\right| \cos \left(\delta_{i}-\delta_{j}-\alpha_{i j}\right), \\
& F_{i j}=3 m_{P} E_{i} E_{j}\left|Y_{i j}\right| \cos \left(\delta_{i}-\delta_{j}-\alpha_{i j}\right) .
\end{aligned}
$$

By lemma 4 , for all $\delta_{i}, \delta_{j} \in \mathcal{I}_{\theta}$ where $i, j \in\{1,2, \ldots, n\}$, there is $\left|\delta_{i}-\delta_{j}\right|<\pi / 2-\alpha_{\max }$, where $\alpha_{\max }=$ $\max _{i j} \tan ^{-1}\left(-G_{i j} / B_{i j}\right)$. The inequality above means that $\cos \left(\delta_{i}-\delta_{j}-\alpha_{i j}\right)>0$. The system matrix $\vec{F}(t)$ therefore satisfies: (a) its off-diagonal elements are nonnegative and (b) its row sums are zero. As a result, $\vec{F}(t)$ is a Metzler matrix with zero row sums for every time instant $t$. All components of $\vec{F}$ are bounded as follows,

$$
\begin{aligned}
& \left|F_{i i}\right| \leq 3 m_{P} \sum_{\substack{j=1 \\
j \neq i}}^{n} E_{i} E_{j}\left|Y_{i j}\right| \leq 3 m_{P} E_{\max }^{2} \sum_{\substack{j=1 \\
j \neq i}}^{n}\left|Y_{i j}\right|, \\
& \left|F_{i j}\right|=3 m_{P} E_{i} E_{j}\left|Y_{i j}\right| \leq 3 m_{P} E_{\max }^{2}\left|Y_{i j}\right| .
\end{aligned}
$$

Under the bound given above and the fact that $\vec{F}(t)$ is Metzler with zero row sums, one can use Theorem 1 in [15] to conclude uniform exponential stability such that all frequencies $\left\{\dot{\delta}_{i}\right\}$ exponentially converge to a common frequency $\dot{\delta}_{\infty}=\omega_{\text {set }}$.

The following theorem establishes the asymptotic voltage stability.

Theorem 4: Assume that conditions in lemma 3 and 4 as well as theorem 2 hold. Define $B_{1}$ and $B_{2}$ as

$$
\begin{aligned}
& B_{1}=\frac{K_{Q}}{m_{Q}}+2 \min _{i}\left(\left(E_{\text {set }, i}+E_{i}\right) Q_{\text {load }, i}\right), \\
& B_{2}=m_{E},
\end{aligned}
$$

where $n$ the number of buses in the network. If

$$
B_{1}>B_{2}
$$

then all voltage magnitudes $\left\{E_{i}\right\}$ asymptotically converge to $\left\{E_{\text {set }, i}\right\}$.

Proof: Taking the derivative of $V_{E}=\sum_{i=1}^{n} \frac{1}{2 m_{Q}} \tilde{E}_{i}^{2}$, we obtain,

$$
\begin{aligned}
\dot{V}_{E}= & \sum_{i=1}^{n} \frac{1}{m_{Q}} \tilde{E}_{i} \dot{\tilde{E}}_{i}, \\
= & \sum_{i=1}^{n}\left\{-\left[\frac{K_{Q}}{m_{Q}}+\left(2 E_{\text {set }, i}+\tilde{E}_{i}\right) Q_{\text {load }, i}\right] \tilde{E}_{i}^{2}\right. \\
& \left.+\tilde{Q}_{i} \tilde{E}_{i}\right\}, \\
= & -\tilde{E}^{T} \operatorname{Diag}\left(\frac{\mathrm{K}_{\mathrm{Q}}}{\mathrm{m}_{\mathrm{Q}}}+\left(2 \mathrm{E}_{\text {set }, \mathrm{i}}+\tilde{\mathrm{E}}_{\mathrm{i}}\right) \mathrm{Q}_{\text {load }, \mathrm{i}}\right) \tilde{\mathrm{E}}+\tilde{\mathrm{Q}}^{\mathrm{T}} \tilde{\mathrm{E}} .
\end{aligned}
$$

Because lemma 4 and theorem 3 imply convergence of $\left\{\delta_{i}\right\}$ to $\left\{\delta_{\text {set }, i}\right\}$, for any $\epsilon_{\delta}$ there is a time $T$ such that when $t>T$ there is $\left|\tilde{\delta}_{i}\right|_{\max }<\epsilon_{\delta}$. Due to lemma $2,\|\tilde{Q}\|_{2}$ is bounded above by $m_{E}\|\tilde{E}\|_{2}+\sqrt{n} m_{\delta} \epsilon_{\delta}$. As a result, the derivative of $V_{E}$ is bounded as,

$$
\begin{aligned}
\dot{V}_{E} \leq & m_{E} \tilde{E}^{T} \tilde{E}+\sqrt{n} m_{\delta} \epsilon_{\delta} \sum_{i=1}^{n}\left|\tilde{E}_{i}\right| \\
& -\tilde{E}^{T} \operatorname{Diag}\left(\frac{\mathrm{K}_{\mathrm{Q}}}{\mathrm{m}_{\mathrm{Q}}}+\left(2 \mathrm{E}_{\text {set }, \mathrm{i}}+\tilde{\mathrm{E}}_{\mathrm{i}}\right) \mathrm{Q}_{\text {load }, \mathrm{i}}\right) \tilde{\mathrm{E}}, \\
= & \tilde{E}^{T} \operatorname{Diag}\left(\mathrm{m}_{\mathrm{E}}-\frac{\mathrm{K}_{\mathrm{Q}}}{\mathrm{m}_{\mathrm{Q}}}-\left(2 \mathrm{E}_{\mathrm{set}, \mathrm{i}}+\tilde{\mathrm{E}}_{\mathrm{i}}\right) \mathrm{Q}_{\text {load }, \mathrm{i}}\right) \tilde{\mathrm{E}} \\
& +\sqrt{n} m_{\delta} \epsilon_{\delta} \sum_{i=1}^{n}\left|\tilde{E}_{i}\right| .
\end{aligned}
$$

There is a subset of $\tilde{E}(t)$ satisfying $\left|\tilde{E}_{i}\right|<2 E_{\text {set }, i}+$ $\left|m_{E}-K_{Q} / m_{Q}+\sqrt{n} m_{\delta} \epsilon_{\delta}\right| /\left|Q_{\text {load }, i}\right|$ for an arbitrary $\epsilon_{\delta}$. Once $\tilde{E}(t)$ enters the subset at $t=T$, it stays in the set thereafter, i.e. the system is uniformly ultimately bounded. As $\epsilon_{\delta}$ goes to zero, $T$ increases and the size of the ultimate bound asymptotically goes to zero. This is sufficient to imply asymptotic convergence of $\tilde{E}$ to zero, which implies voltage stability. As a result, the voltage control block ensures the asymptotic convergence of voltage magnitudes $\left\{E_{i}\right\}$ to the set point $\left\{E_{\text {set }, i}\right\}$.

Remark 4: For voltage magnitude convergence, the ratio $K_{Q} / m_{Q}$ must be large enough to compensate for the reactive loads $\left\{Q_{\text {load }, i}\right\}$.

Remark 5: The reactive loads $\left\{Q_{\text {load }, i}\right\}$ are important for network stability. The allowable disturbance of $Q_{l o a d, i}$ at bus $i$ is restricted by both $m_{E}$ and $K_{Q} / m_{Q}$.

\section{Optimal Dispatch}

The objective of an optimal dispatch problem is to minimize the instantaneous operation cost throughout the network. It is solved by microgrid controllers to minimize the generation cost, subject to power balance relations, constraints on generation capacities, cable power flow limits, voltage and frequency regulation rules, also considering stability constraints. Solutions to this optimization problem are $\left\{P^{*}\right\}$ and $\left\{E^{*}\right\}$, which are fed to CERTS droop controllers.

Given an islanded microgrid with $n$ buses and $m$ links connecting them, with cost functions $C_{P}(\cdot)$ for real power 
generation and $C_{Q}(\cdot)$ for reactive power generation, respectively, this optimal dispatch problem is expressed as

$$
\min C_{P}\left(\sum_{i=1}^{n} P_{g e n, i}\right)+C_{Q}\left(\sum_{i=1}^{n} Q_{g e n, i}\right)
$$

w.r.t. $E_{i}^{*} \quad P_{i}^{*} \quad(i=1,2, \ldots, n)$,

sub. to:for all $i \in\{1,2, \ldots, n\})$ and $j \in\{1,2, \ldots, m\}$

Generation Capacity Limits $\left(\underline{P}_{g e n}, \bar{P}_{g e n}, \underline{Q}_{g e n}, \bar{Q}_{g e n}\right)$

$\underline{P}_{g e n, i} \leq P_{g e n, i} \leq \bar{P}_{g e n, i}, \underline{Q}_{g e n, i} \leq Q_{g e n, i} \leq \bar{Q}_{g e n, i}$,

Power Flow Constraints $\left(\underline{P}_{l n}, \bar{P}_{l n}, \underline{Q}_{l n}, \bar{Q}_{l n}\right)$,

$\underline{P}_{l n, j} \leq P_{l n, j} \leq \bar{P}_{l n, j}, \underline{Q}_{l n, i} \leq Q_{l n, i} \leq \bar{Q}_{l n, i}$,

Voltage and Frequency Regulation Rule $(\underline{E}, \bar{E}, \underline{\omega}, \bar{\omega})$,

$\underline{E}_{i} \leq E_{i} \leq \bar{E}_{i}, \underline{\omega} \leq \omega_{\text {set }} \leq \bar{\omega}$,

Power Balance Relationship (4-5),

Stability Constraints (21-22, 23, 25-26).

An example will be provided in Section VI showing that constraints in equations (21-22, 23, 25-26) ensure stability and the system may be unstable without these constraints.

\section{Simulation EXPERIMENTS}

The schematic diagram of the simulation model is shown in Figure 3. This is an islanded microgrid with three buses, where ideal voltage sources are connected through droop controllers. According to rural electrification scenarios, power generation and load levels are below 10kVA and the voltage level is $480 \mathrm{~V}$. Network links are AWG 6 cables, with an impedance of $(2.5+0.185 j) \Omega /$ mile and a length of one mile. Base values for p.u. normalization are $S_{\text {base }}=10 \mathrm{kVA}$ and $V_{\text {base }}=480 \mathrm{~V}$.

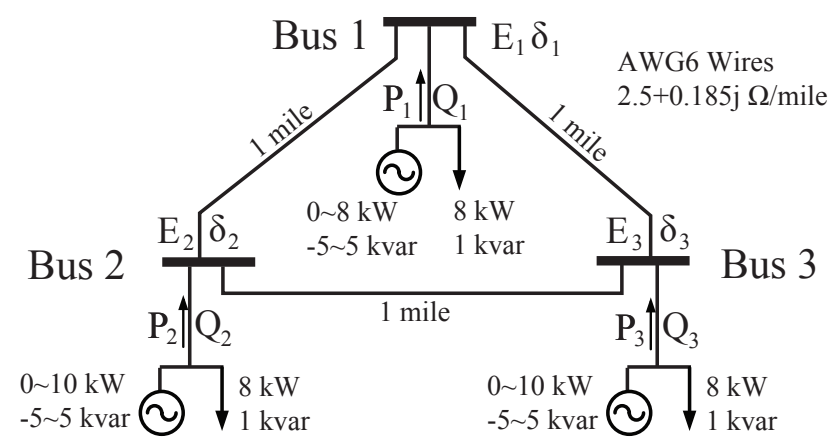

Fig. 3. Simulation Model of an Islanded Microgrid

In this section, a load variation and the response of the controller are simulated. The procedure is as following: the simulation starts from zero initial conditions, known as a "black start"; an equilibrium is reached where each generator supplies its local load, so that no power is injected at bus $i$, i.e. $P_{i}=Q_{i}=0$; two seconds into the simulation, the load on bus 1 increases by $2 \mathrm{~kW}$, while the controller increase the injected power from the other two buses to meet the load.

Simulation tests intend show three aspects: (a) constraints derived in this paper ensure stability and not including them in optimal dispatch problem leads to unstable systems; (b) the constraints are conservative, because of sufficient stability conditions are obtained in this paper; (c) the stability constraints in equations $(21-22,23,25-26)$ make it possible to control extremely weak networks.

Based on equation (16), there must be $K_{Q} / m_{Q}>3061.2$. By selecting $K_{Q}=160$ with $m_{Q}=0.05$, the condition is satisfied. The system is stable and its response on bus 1 is demonstrated in Figure 4.
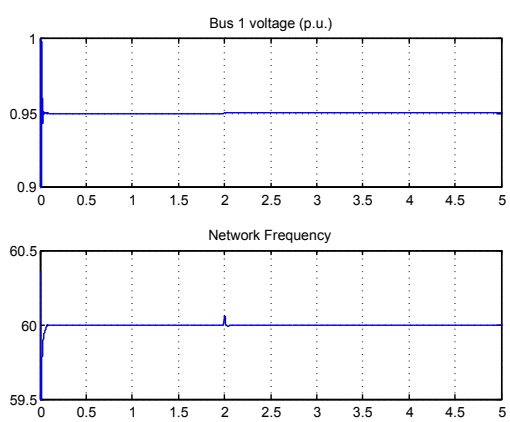

Fig. 4. Simulation Result with $K_{Q} / m_{Q}=3200$

By examining the top plot in Figure 4, one sees that the voltage just prior to $t=2 \mathrm{sec}$ is a constant 0.95 p.u.. After the load change, the voltage exhibits a temporary increase of less than 0.0002 p.u., which corresponds to a $0.02 \%$ voltage ripple. The bottom plot shows the frequency in the network. Within $0.15 \mathrm{sec}$ after the load change, the network frequency changes by a maximum of $0.05 \mathrm{~Hz}$. Thereafter, the frequency converges to the desired value $60 \mathrm{~Hz}$.

To demonstrate that the stability constraints are essential to ensure system stability, a dispatch problem without those constraints is solved. The controller parameter $K_{Q}$ is chosen to be one, with $m_{Q}=0.05$, the ratio $K_{Q} / m_{Q}=20$. As shown in Figure 5, the system shows instabilities in both voltage and frequency signals.

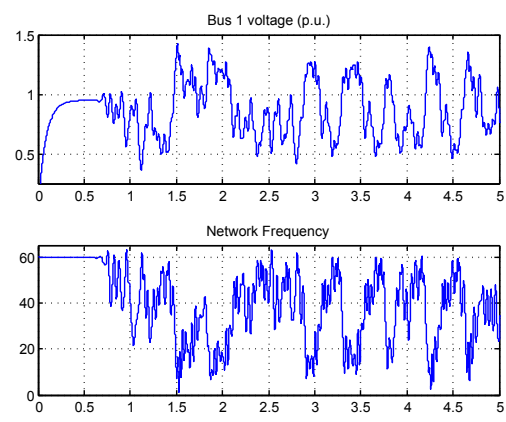

Fig. 5. Simulation Result with $K_{Q} / m_{Q}=20$

Figure 5 shows that even before the load changes, the system is unstable. In the top plot, one sees that the voltage deviate from the nominal value by more than $50 \%$. In the bottom plot, the network frequency decreases below $20 \mathrm{~Hz}$. Even though the signals are bounded, they are deemed as unstable in a power network. As a result, the stability 
constraints are critical to ensure stability in the optimal dispatch problems.

The ratio $K_{Q} / m_{Q}$ demonstrates the control force of the inverter, physically. Reducing this ratio may bring benefits when these inverters are actually built. It is shown in this simulation that, with a reduced $K_{Q} / m_{Q}$, the system still has voltage stability and frequency synchronization. As demonstrated in Figure 6, simulations are conducted with $K_{Q} / m_{Q}=300$ (left) and 200(right), respectively.
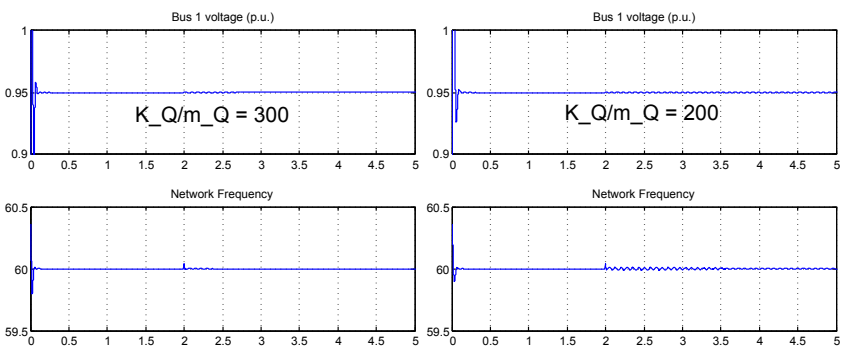

Fig. 6. Simulation Results with $K_{Q} / m_{Q}=300$ (left) and 200(right)

Figure 6 shows that when the ratio $K_{Q} / m_{Q}$ is reduced to about $1 / 10$ (when $K_{Q} / m_{Q}=300$ ) of the limit given in equation (16), the system still has voltage stability and frequency synchronization. However, the voltage magnitude at bus 1 has a ripple of $0.05 \%$ and the system frequency sees a ripple of $0.01 \mathrm{~Hz}$. Further reduce the ratio to 200, as shown in the right plots of Figure 6, the amplitudes of the ripples in voltage magnitude and the system frequency are basically the same. The difference is that, with a smaller control force, the signals converge slower in this case. Because the constraints derived in this paper are only sufficient conditions of system stability, the conservativeness demonstrated here is predictable.

Although AWG 6 cables $(4.11 \mathrm{~mm}$ in diameter) is small for low-voltage distribution networks, even thinner cables may be applied in rural electrification projects, such as AWG 16 cables $(1.29 \mathrm{~mm}$ in diameter) used to connect solar panels. These cables are assumed to have roughly ten times of resistance and the same reactance as AWG 6 cables, i.e. $(25+0.185 j) \Omega /$ mile. Based on equation (16), there must be $K_{Q} / m_{Q}>292.5$. By selecting $K_{Q}=15$ with $m_{Q}=0.05$, the condition is satisfied. The system is stable and its response on bus 1 is demonstrated in Figure 7.
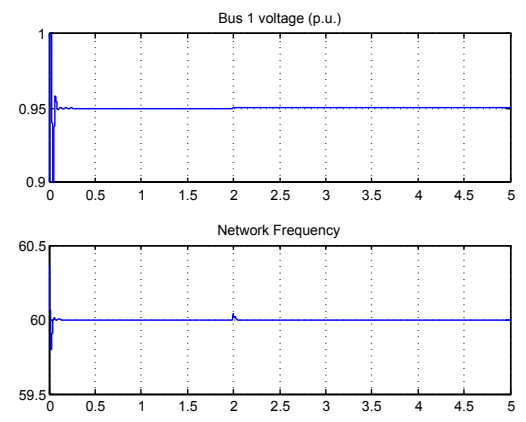

Fig. 7. Weaker Network Simulation with $K_{Q} / m_{Q}=300$
By examining the top plot in Figure 7, one sees that the voltage just prior to $\mathrm{t}=2 \mathrm{sec}$ is a constant 0.95 p.u.. After the load change, the voltage exhibits a temporary increase of less than 0.0001p.u., which corresponds to a $0.01 \%$ voltage ripple. The bottom plot shows the frequency in the network. Within $0.1 \mathrm{sec}$ after the load change, the network frequency changes by a maximum of $0.04 \mathrm{~Hz}$. Thereafter, the frequency converges to the desired value $60 \mathrm{~Hz}$. As a result, the stability constraints make it possible to control extremely weak networks.

In this section, simulation tests are conducted to the islanded microgrid model. Based on the simulation results, we can conclude that constraints derive in this paper ensures stability and not including them in optimal dispatch problems leads to unstable systems. Because the stability conditions in this paper are only sufficient ones, the constraints derived are somewhat conservative, which means a system not satisfying these constraints may still be stable. Furthermore, it is shown that the stability constraints in equations $(21-22,23,25-$ 26) make it possible to control extremely weak networks, this property is especially valuable for rural electrification projects.

\section{Summary AND Future WORK}

Microgrids represent a bottom-up approach for improving the power security of critical loads that cannot tolerate disruptions in main grid service. Voltage stability becomes a significant issue in microgrids when the network interconnections are weak. This paper derives sufficient conditions for voltage stability of a weak microgrid with inverter connected sources. These conditions take the form of inequality constraints on various network parameters, loads and generation setpoints. These conditions can therefore be easily incorporated into dispatch optimization problems.

Future research will focus on the optimal control of an islanded microgrid or coupled microgrids, where stability constraints $(21-22,23,25-26)$ have been added to the constrained optimal dispatch problem in Section V. This represents a point wise constraint that could be viewed as a model predictive control (MPC) scheme with a horizon length of zero [17]. Results in this paper will be extended to a true MPC controller similar to that used in [10] and these MPC problems can be solved in a distributed manner, similar to our earlier work in [19].

\section{APPENDIX}

Proof: With the help of its Jacobian $\frac{\partial Q}{\partial E}$ and $\frac{\partial Q}{\partial \delta}, \tilde{Q}$ is bounded by a function of $\tilde{E}$ and $\tilde{\delta}$. With infinite vector norm and its induced infinite matrix norm, the linearization induces

$$
Q-Q_{\text {set }}=\left.\frac{\partial Q}{\partial E}\right|_{\text {set }}\left(E-E_{\text {set }}\right)+\left.\frac{\partial Q}{\partial \delta}\right|_{\text {set }}\left(\delta-\delta_{\text {set }}\right) .
$$

Taking infinite vector norms on both sides, there is

$$
\left\|Q-Q_{\text {set }}\right\|_{\infty} \leq\left\|\frac{\partial Q}{\partial E}\left(E-E_{\text {set }}\right)+\frac{\partial Q}{\partial \delta}\left(\delta-\delta_{\text {set }}\right)\right\|_{\infty} .
$$


Taking infinite vector norms on both sides, there is

$$
\begin{aligned}
\|\tilde{Q}\|_{\infty} & \leq\left\|\frac{\partial Q}{\partial E} \tilde{E}+\frac{\partial Q}{\partial \delta} \tilde{\delta}\right\|_{\infty}, \\
& \leq\left\|\frac{\partial Q}{\partial E} \tilde{E}\right\|_{\infty}+\left\|\frac{\partial Q}{\partial \delta} \tilde{\delta}\right\|_{\infty}, \\
& \leq\left\|\frac{\partial Q}{\partial E}\right\|_{\infty}\|\tilde{E}\|_{\infty}+\left\|\frac{\partial Q}{\partial \delta}\right\|_{\infty}\|\tilde{\delta}\|_{\infty}, \\
\left|\tilde{Q}_{i}\right|_{\max } & \leq\left\|\frac{\partial Q}{\partial E}\right\|_{\infty}\left|\tilde{E}_{i}\right|_{\max }+\left\|\frac{\partial Q}{\partial \delta}\right\|_{\infty}\left|\tilde{\delta}_{i}\right|_{\max },
\end{aligned}
$$

where $\left\|\frac{\partial Q}{\partial E}\right\|_{\infty}$ and $\left\|\frac{\partial Q}{\partial \delta}\right\|_{\infty}$ are bounded as following

$$
\begin{aligned}
& \left\|\frac{\partial Q}{\partial E}\right\|_{\infty}=3 \sum_{\substack{j=1 \\
j \neq i}}\left|E_{i}\left(G_{i j} \sin \left(\delta_{i}-\delta_{j}\right)-B_{i j} \cos \left(\delta_{i}-\delta_{j}\right)\right)\right| \\
& +\left|3 \sum_{\substack{j=1 \\
j \neq i}} E_{i}\left(G_{i j} \sin \left(\delta_{i}-\delta_{j}\right)-B_{i j} \cos \left(\delta_{i}-\delta_{j}\right)\right)-6 E_{i} B_{i i}\right|, \\
& \leq\left|6 E_{i} B_{i i}\right|+6 \sum_{\substack{j=1 \\
j \neq i}}\left|E_{i}\left(G_{i j} \sin \left(\delta_{i}-\delta_{j}\right)-B_{i j} \cos \left(\delta_{i}-\delta_{j}\right)\right)\right|, \\
& \leq 6\left|E_{i}\right|_{\max }\left|B_{i i}\right|_{\max }+6\left|E_{i}\right|_{\max } \max _{i}\left(\sum_{\substack{j=1 \\
j \neq i}}\left(\left|G_{i j}\right|+\left|B_{i j}\right|\right)\right), \\
& \leq 6\left(\max _{i} E_{s e t, i}+\left|\tilde{E}_{i}\right|_{\max }\right)\left(\left|G_{i i}\right|_{\max }+2\left|B_{i i}\right|_{\max }\right)=l_{E} \\
& \left\|\frac{\partial Q}{\partial \delta}\right\|_{\infty} \\
& =\left|3 \sum_{\substack{j=1 \\
j \neq i}} E_{i} E_{j}\left(G_{i j} \cos \left(\delta_{i}-\delta_{j}\right)+B_{i j} \sin \left(\delta_{i}-\delta_{j}\right)\right)\right| \\
& +3 \sum_{j=1}\left|E_{i} E_{j}\left(G_{i j} \cos \left(\delta_{i}-\delta_{j}\right)+B_{i j} \sin \left(\delta_{i}-\delta_{j}\right)\right)\right|, \\
& j \neq i \\
& \leq 6 \sum_{\substack{j=1 \\
j \neq i}}\left|E_{i} E_{j}\left(G_{i j} \cos \left(\delta_{i}-\delta_{j}\right)+B_{i j} \sin \left(\delta_{i}-\delta_{j}\right)\right)\right|, \\
& \leq 6\left|E_{i}\right|_{\max }^{2} \max _{i}\left(\sum_{\substack{j=1 \\
j \neq i}}\left(\left|G_{i j}\right|+\left|B_{i j}\right|\right)\right), \\
& \leq 6\left(\max _{i} E_{s e t, i}+\left|\tilde{E}_{i}\right|_{\max }\right)^{2}\left(\left|G_{i i}\right|_{\max }+\left|B_{i i}\right|_{\max }\right)=l_{\delta} \text {. }
\end{aligned}
$$

Proof: If system frequency synchronizes and phase shifts are within the invariant set $\mathcal{I}_{\theta}$, then for any $i, j \in$ $\{1,2, \ldots, n\}$ there is $\left|\delta_{i}-\delta_{j}\right| \leq \alpha_{\max }$. Within the invariant set $\mathcal{I}_{E}$, applying vector two-norm and its induced matrix two-norm, there is

$$
\begin{aligned}
\|\tilde{Q}\|_{2} & =\left\|\frac{\partial Q}{\partial E} \tilde{E}+\frac{\partial Q}{\partial \delta} \tilde{\delta}\right\|_{2} \\
& \leq\left\|\frac{\partial Q}{\partial E} \tilde{E}\right\|_{2}+\left\|\frac{\partial Q}{\partial \delta} \tilde{\delta}\right\|_{2} \\
& \leq\left\|\frac{\partial Q}{\partial E}\right\|_{2}\|\tilde{E}\|_{2}+\left\|\frac{\partial Q}{\partial \delta}\right\|_{2} \sqrt{n}\left|\tilde{\delta}_{i}\right|_{\max } \\
& \leq m_{E}\|\tilde{E}\|_{2}+\sqrt{n} m_{\delta}\left|\tilde{\delta}_{i}\right|_{\max }
\end{aligned}
$$

\section{REFERENCES}

[1] J.A. Acebron, L.L. Bonilla, C.J.P. Bicente, Felix Ritort and Renato Spigler. The Kuramoto Model: a Simple Paradigm for Synchronization Phenomena. Reviews of Modern Physics, vol.77, Jan. 2005.

[2] F.L. Alvarado, J. Meng, C.L. DeMarco and W.S. Mota. Stability analysis of interconnected power systems coupled with market dynamics. Power Systems, IEEE Transactions on, vol.16, no.4, pp.695-701, Nov 2001.

[3] D. Casagrande, A. Astolfi and R. Ortega. Global stabilization of non-globally linearizable triangular systems: Application to transient stability of power systems. Decision and Control and European Control Conference (CDC-ECC), 2011 50th IEEE Conference on , vol., no., pp.331-336, 12-15 Dec. 2011.

[4] K. De Brabandere, B. Bolsens, J. Van den Keybus, A. Woyte, J. Driesen and R. Belmans. A Voltage and Frequency Droop Control Method for Parallel Inverters. Power Electronics, IEEE Transactions on , vol.22, no.4, pp.1107-1115, July 2007.

[5] F. Dorfler and F. Bullo. On the Critical Coupling for Kuramoto Oscillators. SIAM Journal on Applied Dynamical Systems, 10(3):1070-1099, 2011.

[6] F. Dorfler and F. Bullo. On the critical coupling strength for Kuramoto oscillators. American Control Conference (ACC), vol., no., 2011 , pp.3239-3244, June 29 2011-July 12011.

[7] F. Dorfler and F. Bullo. Synchronization and Transient Stability in Power Networks and Non-Uniform Kuramoto Oscillators. SIAM Journal on Control and Optimization, 50(3):1616-1642, 2012.

[8] I.A. Engler. Applicability of Droops in Low Voltage Grids. International Journal of Distributed Energy Resources, vol.1 no.1, Jan-Mar. 2005.

[9] F.D. Galiana. Analytical Investigation of the Power Flow Equations. American Control Conference, 1983 , vol., no., pp.411-415, 22-24 June 1983.

[10] I.A. Hiskens and B. Gong. MPC-Based Load Shedding for Voltage Stability Enhancement. Decision and Control, 2005 and 2005 European Control Conference. CDC-ECC '05. 44th IEEE Conference on , vol., no., pp. 4463- 4468, 12-15 Dec. 2005.

[11] M. Illindala and G. Venkataramanan. Small Signal Stability of a Microgrid with Parallel Connected Distributed Generation. Intellegent Automation and Soft Computing, vol.16, no.2, pp.231-250, 2010.

[12] S.V. Iyer, M.N. Belur and M.C. Chandorkar. A Generalized Computational Method to Determine Stability of a Multi-inverter Microgrid. Power Electronics, IEEE Transactions on , vol.25, no.9, pp.2420-2432, Sept. 2010.

[13] R.H. Lasseter. Smart Distribution: Coupled Microgrids. Proceedings of the IEEE, vol.99, no.6, pp.1074-1082, June 2011.

[14] R. Majumder, G. Ledwich, A. Ghosh, S. Chakrabarti and F. Zare. Droop Control of Converter-Interfaced Microsources in Rural Distributed Generation. Power Delivery, IEEE Transactions on , vol.25, no.4, pp.2768-2778, Oct. 2010.

[15] L. Moreau. Stability of continuous-time distributed consensus algorithms. Decision and Control, 2004. CDC. 43rd IEEE Conference on , vol.4, no., pp. 3998- 4003 Vol.4, 14-17 Dec. 2004.

[16] N. Pogaku, M. Prodanovic and T.C. Green. Modeling, Analysis and Testing of Autonomous Operation of an Inverter-Based Microgrid Power Electronics, IEEE Transactions on , vol.22, no.2, pp.613-625, March 2007.

[17] J.A. Primbs, V. Nevistic and J.C. Doyle. A receding horizon generalization of pointwise min-norm controllers. Automatic Control, IEEE Transactions on , vol.45, no.5, pp.898-909, May 2000.

[18] S. Tabatabaee, H.R. Karshenas, A. Bakhshai and P. Jain. Investigation of droop characteristics and $\mathrm{X} / \mathrm{R}$ ratio on small-signal stability of autonomous Microgrid. Power Electronics, Drive Systems and Technologies Conference (PEDSTC), 2011 2nd , vol., no., pp.223-228, 16-17 Feb. 2011.

[19] Pu Wan and M.D. Lemmon. Optimal power flow in microgrids using event-triggered optimization. American Control Conference (ACC), 2010 , vol., no., pp.2521-2526, June 30 2010-July 22010.

[20] Yang Wang, Zongxiang Lu, Yong Min and Zhong Wang. Small signal analysis of microgrid with multiple micro sources based on reduced order model in islanding operation. Power and Energy Society General Meeting, 2011 IEEE, vol., no., pp.1-9, 24-29 July 2011.

[21] G. Venkataramanan and M. Illindala. Small Signal Dynamics of Inverter Interfaced Distributed Generation in a Chain-Microgrid. Power Engineering Society General Meeting, 2007. IEEE , vol., no., pp.1-6, 24-28 June 2007. 